\title{
Stability Analysis of Mathematical Model for New Hydraulic Bilateral Rolling Shear
}

\author{
QingXue HUANG, ${ }^{1)}$ Jia LI, ${ }^{1)}$ HongZhou $\mathrm{LI}^{2)} \mathrm{HeYong}_{\mathrm{HAN}}{ }^{1 / *}$ and LiDong MA ${ }^{11}$ \\ 1) Heavy Machinery Eng. Research Center of Education Ministry, Taiyuan Univ. of Sci. and Technol., Taiyuan, 030024 PR China. \\ 2) Hebei Wenfeng Iron and Steel Co. Ltd, Hebei, Wuan, PR China.
}

(Received on June 17, 2015; accepted on November 25, 2015)

\begin{abstract}
In this study, the model of new hydraulic bilateral rolling shear is researched based on kinematics theory of a hybrid spatial linkage mechanism. Combined with research method of 3-RRR planar parallel mechanisms kinematic, the relationship between cylinder displacements and blade positions of the shearing mechanism is developed. Nonlinear system stability of the shearing mechanism in a new hydraulic bilateral rolling shear is researched based on Krasovskii method, the result shown that the system is stable and reliable. Moreover, based on the bilateral rolling shear of a large steel company, the displacement of cylinders is obtained through experiments. Compared with differential between the theoretical formula and the actual value, the differential is small. This study has certain instruction to the design and the development of similar product and it also has a higher practical value to the general industrial production.
\end{abstract}

KEY WORDS: spatial linkage mechanism; new hydraulic bilateral rolling shear; mathematic model; Krasovskii method.

\section{Introduction}

A bilateral rolling shear for cutting the width of a medium steel plate in a production line while simultaneously shearing both sides of the plate via the stepping cut method ${ }^{1,2)}$ is designed as shown in Fig. 1. A mechanical bilateral rolling shear generally applies to the complex three-shaft tri-eccentric architecture and inclined-angle shear ${ }^{3,4)}$ (Fig. 2) and their requirement for synchronous cutting control are extremely high. And the equipment maintenance is difficult. As shown in Fig. 2, Horizontal component $F_{2}$ acted on the steel plate produced from upper blade increase the friction of the cross-section, which lead to some built-up edges. Inclined-angle shear with an additional large travel, which causing serious collapse angle in the cut end of plate as shown in Fig. 3. However, the arcuate blade has no sideto-side motion in the process of shearing for new hydraulic bilateral rolling shear. In stable generating phase for the new hydraulic rolling shear, the actual effective area of the steel sheet is limited to the shaded $\mathrm{ACD}, \mathrm{P}_{1}$ is the maximum shear force what happened on the area in the process of rollcutting $^{5)}$ as shown in Fig. 4. Due to the role of the guide rod HG in Fig. 5, the center of gravity of the blade does not swing during cutting process, which ensures good quality of section. Friction force between the upper blade and plate is reduced so as to prolong the service life. Amount of scissors overlapping is held constant, which can guarantee that the curvature, levelness and the final cut shape of cutting end of the medium heavy steel plates are appropriate. The

\footnotetext{
*Corresponding author: E-mail: mkj_lj@126.com

DOI: http://dx.doi.org/10.2355/isijinternational.ISIJINT-2015-324
}

shearing mechanism of the new hydraulic bilateral rolling shear uses servo cylinders, which are positioned horizontally on both sides of the frame to drive the rods while the upper shear blade rolling cut the plate. It has a good effect on reinforcement and the minimum swinging angle of cylinders. As shown in reference, ${ }^{6)}$ the larger force which can cut through the steel plate may be produced by smaller thrust of hydraulic cylinder and the thrust forces depend on the load, so the device has a simple structure, a large shearing force,

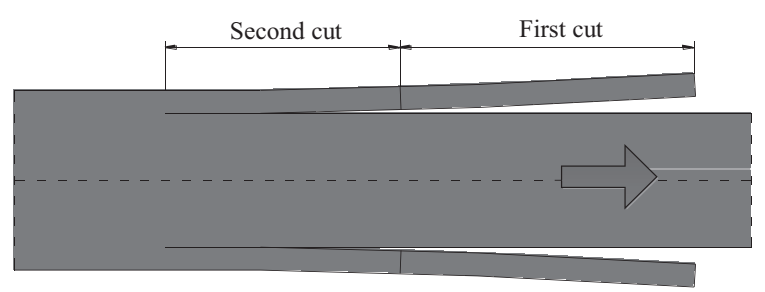

Fig. 1. Cutting steps demonstration of the rolling cut of the bilateral shear.

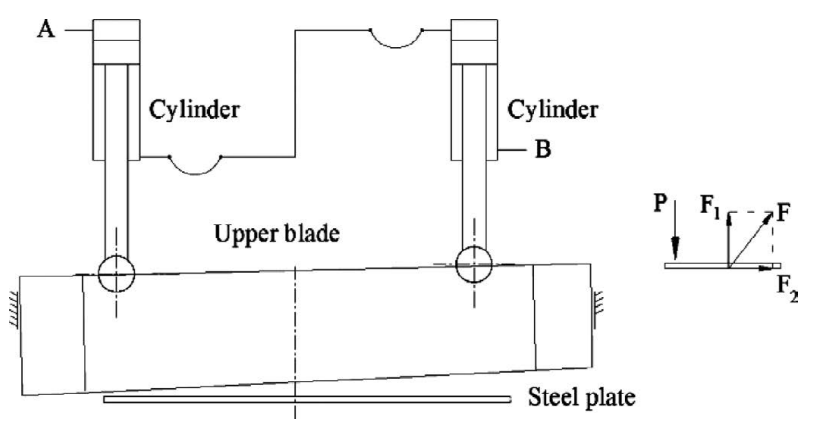

Fig. 2. The structure of hydraulic inclined-angle shear and force diagram at cutting shear. 
and high control accuracy. The new shearing mechanism has more advantage than the conventional in the quality of the cross section of plate steel. At present, a prototype of the device has been developed and applied in a production test. Hence, the reliability of the shearing mechanism should be further studied.

Nowadays, mathematical model on spatial shear mechanism optimization of steel rolling shear is established and applied for some large Iron \& Steel plant. ${ }^{7)}$ Based on the establishment of composite linkage position loop equation, moment balance equation and hydraulic control system model is calculated. ${ }^{8)}$ Above literatures studied bilateral rolling shear only based on the simulation test and verify, but there is no in-depth research in theory for the shearing mechanism. The motion of mechanism of new hydraulic bilateral rolling shear is complex and difficult, which bring a great deal of difficulty in researching the analysis theory

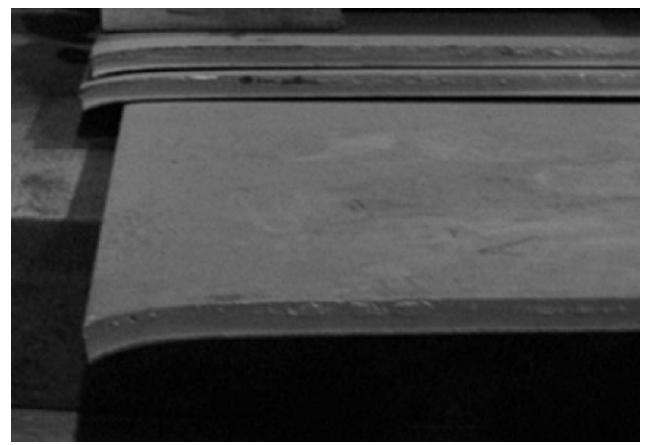

Fig. 3. Collapsed angle default by inclined-angle shear.

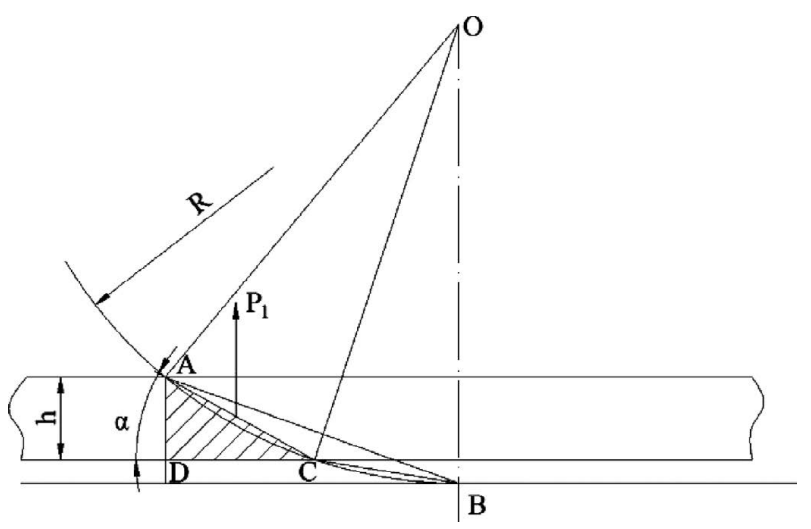

Fig. 4. Sketch map of shear mechanics model.

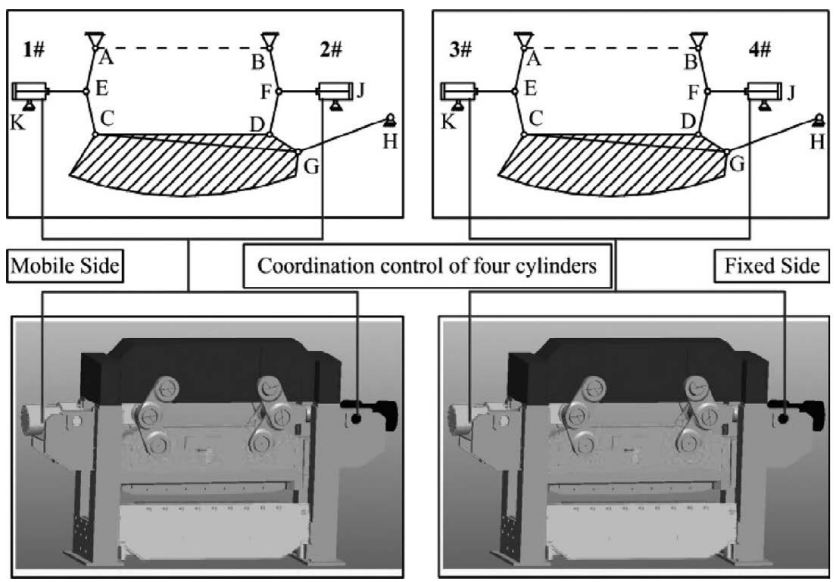

Fig. 5. New hydraulic bilateral rolling shear. of mechanism dynamic. Different from the traditional geometric construction, the motion way of different institutions of shear mechanism need to be acted as the given track. The upper blade of the hydraulic bilateral rolling shear exhibits rolling shear movement, which is complex. The displacement of the upper blade cannot be directly measured, and there is no a set of accurate calculation method about the position and posture of the upper blade. Moreover, analyzing the mechanism motion is attracted attention by so many scholars in different periods. Approximate integrated approach developed in recent years is rising, a comprehensive method used is diversity, but the comprehensive theoretical study has not been perfect, there are still some limitations on the method.

Based on kinematics theory of a hybrid spatial linkage mechanism, combined with research method of 3-RRR planar parallel mechanisms kinematic, the mathematical model between the displacements of four hydraulic servo cylinders and the position of the upper blade is developed and the stability of shearing mechanism is put forward based on the Krasovskii method. Moreover, it provides a theoretical basis for the nonlinear coupling control of nonlinear multi-DOF systems and new theories and methods is developed for the kinematics and dynamic analysis of the mechanism.

\section{Building the Mathematical Model of the Shearing Mechanism of the Bilateral Rolling Shear}

The new hydraulic bilateral rolling shear mainly consists of fixed and mobile scissors (Fig. 5). The beginning and end of the cutting stage is driven only by a single cylinder, in which the movement of the upper blade is simple. This study aims to analyze the action of two hydraulic servo cylinders during the shearing process and to establish the relationship between the position of the upper blade and the displacement of the hydraulic cylinders.

The mobile shearing mechanism is fixed with an appropriate coordinate system $\mathrm{x}, \mathrm{y}$, and $\mathrm{z}$, which is assumed to be a right-handed Cartesian space with an origin point that is coincident with the position of $A$ in the frame (shown in Fig. 6). The angle between the connecting $\operatorname{rod} A E$ and the $\mathrm{x}$ axis is designed as $\theta_{1}$. The angle between the connecting $\operatorname{rod} B F$ and the $\mathrm{x}$ axis is designed as $\theta_{2}$. The angle between

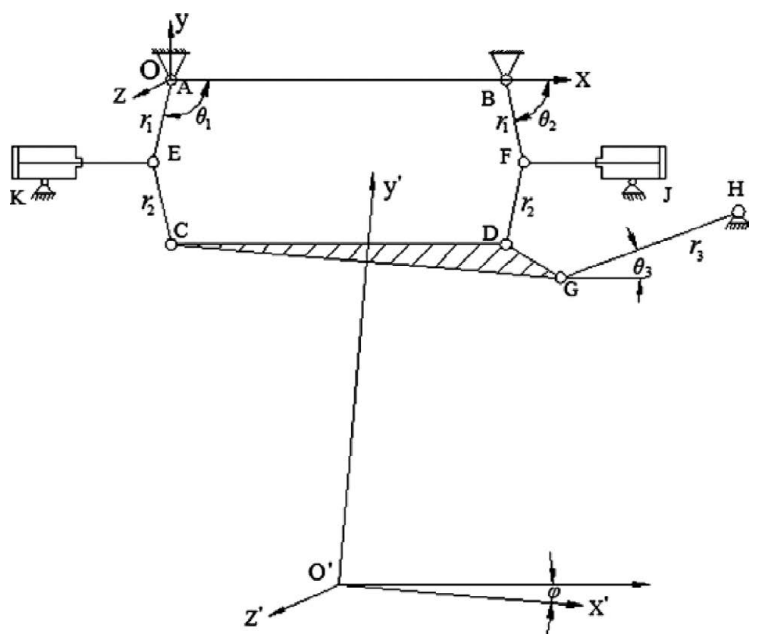

Fig. 6. Agency coordinates of the mobile mechanism. 
the guide $\operatorname{rod} H G$ and the $\mathrm{x}^{\prime}$ axis is designed as $\theta_{3}$. The counterclockwise direction is specified as the positive direction. The translational coordinate system is inclined at angle $\varphi$ toward the direction of the absolute coordinate system.

The coordinates of the hinge point is designed as $A\left(a_{x}\right.$, $\left.a_{y}\right), B\left(b_{x}, b_{y}\right), C\left(c_{x}, c_{y}\right), D\left(d_{x}, d_{y}\right), E\left(e_{x}, e_{y}\right), F\left(f_{x}, f_{y}\right), G\left(g_{x}\right.$, $\left.g_{y}\right)$, and $H\left(h_{x}, h_{y}\right)$. The hinge points of the upper blade attachment form a triangle with the following side lengths: $l_{\mathrm{cd}}=2300 \mathrm{~mm}, l_{\mathrm{dg}}=845 \mathrm{~mm}$, and $l_{\mathrm{cg}}=3048 \mathrm{~mm}$. The coordinate transformation matrix ${ }^{9-11)} \mathrm{T}$ is presented as follows:

$$
\left[\begin{array}{ccc}
\cos \varphi & \sin \varphi & x \\
-\sin \varphi & \cos \varphi & y \\
0 & 0 & 1
\end{array}\right]
$$

Where $x, y$, and $\varphi$ indicate the position of the moving coordinate system $\mathrm{O}^{\prime}$ based on the static coordinate system $\mathrm{O}$.

The coordinates of $C, D$ and $G$ can be expressed as follows:

$$
\left[\begin{array}{ccc}
C & D & G \\
1 & 1 & 1
\end{array}\right]=T\left[\begin{array}{ccc}
C^{\prime} & D^{\prime} & G^{\prime} \\
1 & 1 & 1
\end{array}\right]
$$

The coordinates of all the joints of the upper blade based on the moving coordinate system $\mathrm{O}^{\prime}$ are $\mathrm{C}^{\prime}(-1521,2315)$, $\mathrm{D}^{\prime}(752,2665), \mathrm{G}^{\prime}(1521,2315)$ and $\mathrm{H}(4$ 251, - 1465$)$. Then, the transformed matrix is presented as follows:

$$
\left[\begin{array}{ccc}
C_{x}^{\mathrm{I}} & D_{x}^{\mathrm{I}} & G_{x}^{\mathrm{I}} \\
C_{y}^{\mathrm{I}} & D_{y}^{\mathrm{I}} & G_{y}^{\mathrm{I}} \\
1 & 1 & 1
\end{array}\right]=\left[\begin{array}{ccc}
\cos \varphi & \sin \varphi & x \\
-\sin \varphi & \cos \varphi & y \\
0 & 0 & 1
\end{array}\right]\left[\begin{array}{ccc}
-1521 & 752 & 1521 \\
2315 & 2665 & 2315 \\
1 & 1 & 1
\end{array}\right]
$$

The starting angle between the moving coordinate system and the static coordinate system is $\varphi=6.37^{\circ}$.

Then, the position coordinates of $E$ and $F$ are given as follows:

$$
\begin{aligned}
& E_{x}^{\mathrm{I}}=C_{x}^{\mathrm{I}}+r_{2} \cos \alpha, \quad E_{y}^{\mathrm{I}}=C_{y}^{\mathrm{I}}+r_{2} \sin \alpha \\
& F_{x}^{\mathrm{I}}=D_{x}^{\mathrm{I}}+r_{2} \cos \beta, \quad F_{y}^{\mathrm{I}}=D_{y}^{\mathrm{I}}+r_{2} \sin \beta
\end{aligned}
$$

Where $r_{2}=l_{\mathrm{ce}}=l_{\mathrm{df}}=702 \mathrm{~mm}, \alpha, \beta$ are any angle of trigonometric function.

The displacements of $E$ obtained from Eq. (4) in the horizontal and vertical directions are respectively given as follows:

$$
\begin{aligned}
& \Delta E_{x}^{\mathrm{I}}=-1521 \cos \varphi+2315 \sin \varphi+1255, \\
& \Delta E_{y}^{\mathrm{I}}=1521 \sin \varphi+2315 \cos \varphi-2469
\end{aligned} .
$$

Where $\Delta E_{x}^{\mathrm{I}}$ and $\Delta E_{y}^{\mathrm{I}}$ are the horizontal and vertical displacements, respectively, of 1 \# cylinder.

The displacements of $F$ obtained from Eq. (5) in the horizontal and vertical directions are respectively given as follows:

$$
\begin{aligned}
& \Delta F_{x}^{\mathrm{I}}=752 \cos \varphi+2665 \sin \varphi-1043, \\
& \Delta F_{y}^{\mathrm{I}}=-752 \cos \varphi+2665 \sin \varphi-2565
\end{aligned}
$$

Where $\Delta F_{x}^{\mathrm{I}}$ and $\Delta F_{y}^{\mathrm{I}}$ are the horizontal and vertical displacements, respectively, of $2 \#$ cylinder.

The displacements of $G$ obtained from Eq. (3) in the horizontal and vertical directions are respectively given as follows:

$$
\begin{aligned}
& \Delta G_{x}^{\mathrm{I}}=1521 \cos \varphi+2315 \sin \varphi-1768, \\
& \Delta G_{y}^{\mathrm{I}}=-1521 \sin \varphi+2315 \cos \varphi-2132
\end{aligned}
$$

Where $\Delta G_{x}^{\mathrm{I}}$ and $\Delta G_{y}^{\mathrm{I}}$ are the horizontal and vertical displacements of point $G$, respectively.

In addition, $E, F$, and $G$ exhibit a circular motion at the centers of $A, B$, and $H$, respectively. The coordinates of $E$, $F$, and $G$ are respectively given as follows:

$$
\begin{array}{r}
E_{x}^{\mathrm{II}}=r_{1} \cos \theta_{1}, \quad E_{y}^{\mathrm{II}}=r_{1} \sin \theta_{1} \ldots \ldots \\
F_{x}^{\mathrm{II}}=r_{1} \cos \theta_{2}+2300, \quad F_{y}^{\mathrm{II}}=r_{1} \sin \theta_{2} \\
G_{x}^{\mathrm{II}}=-r_{3} \cos \theta_{3}+4251, \quad G_{y}^{\mathrm{II}}=-r_{3} \sin \theta_{3}-1465
\end{array}
$$

Where $r_{3}=l_{\mathrm{gh}}=1260 \mathrm{~mm}, r_{1}=l_{\mathrm{ea}}=l_{\mathrm{bf}}=702 \mathrm{~mm}, \theta_{1}=-108^{\circ}$, $\theta_{2}=-65^{\circ}, \theta_{3}=9.71^{\circ}$, and $\varphi=6.37^{\circ}$.

Where the displacements of $E, F$, and $G$ obtained from Eqs. (9), (10), and (11) in the horizontal and vertical directions are respectively given as follows:

$$
\begin{aligned}
& \Delta E_{x}^{\mathrm{II}}=702 \cos \theta_{1}+217, \quad \Delta E_{y}^{\mathrm{II}}=702 \sin \theta_{1}+668 \ldots \ldots \ldots \\
& \Delta F_{x}^{\mathrm{II}}=702 \cos \theta_{2}-297, \quad \Delta F_{y}^{\mathrm{II}}=702 \sin \theta_{2}+636 \ldots \ldots . . \\
& \Delta G_{x}^{\mathrm{II}}=-1260 \cos \theta_{3}+1242, \Delta G_{y}^{\mathrm{II}}=-1260 \sin \theta_{3}+213 \ldots \ldots
\end{aligned}
$$

Where $\Delta E_{x}^{\mathrm{II}}$ and $\Delta E_{y}^{\mathrm{II}}$ are the horizontal and vertical displacements, respectively, of $1 \#$ cylinder; $\Delta F_{x}^{\mathrm{II}}$ and $\Delta F_{y}^{\mathrm{II}}$ are the horizontal and vertical displacements, respectively, of $2 \#$ cylinder; $\Delta G_{x}^{\mathrm{II}}$ and $\Delta G_{y}^{\mathrm{II}}$ are the horizontal and vertical displacements, respectively, of point $G$.

In the preceding equations, $E_{x}=E_{x}^{\mathrm{I}}=E_{x}^{\mathrm{II}}, E_{y}=E_{y}^{\mathrm{I}}=E_{y}^{\mathrm{II}}$.

Solve the equation group as follows to obtain the $\varphi$ value:

$$
\varphi=\arcsin \frac{\cos \theta_{1}-1.48}{3.95}+33.3^{\circ}
$$

The upper blade is rigid, in which the movement of any point are the same during the rolling-cut process. Thus, hinged point $G$ is used to analyze the motion of the upper blade.

The relationship between cylinder displacement and the position of the upper blade can be expressed as follows:

$$
\begin{aligned}
& y_{1}=\sqrt{\Delta E_{x}^{2}+\Delta E_{y}{ }^{2}}=702 \sqrt{2\left(1+\sin \left(\theta_{1}+18^{\circ}\right)\right)} \ldots \\
& y_{2}=\sqrt{\Delta F_{x}^{2}+\Delta F_{y}^{2}}=702 \sqrt{2\left(1+\sin \left(\theta_{2}-25^{\circ}\right)\right)} .
\end{aligned}
$$

$$
\left[\begin{array}{c}
S_{x} \\
S_{y} \\
\sin \varphi \\
\cos \varphi
\end{array}\right]=\frac{1}{492804}\left[\begin{array}{ll}
348.4 & -32.9 \\
45.5 & 349.3 \\
0.1 & -0.08 \\
0.08 & 0.1
\end{array}\right]\left[\begin{array}{l}
y_{1}^{2} \\
y_{2}^{2}
\end{array}\right]+\left[\begin{array}{c}
0 \\
0 \\
0.11 \\
0.99
\end{array}\right]
$$

Where $S_{x}$ and $S_{y}$ are the horizontal and vertical displacements of upper blade for mobile scissors, respectively; and $y_{1}$ and $y_{2}$ are the displacements of $1 \#$ and $2 \#$ hydraulic cylinders for the mobile scissors, respectively.

The relationship between the displacement of the cylinders and the position of the upper blade for mobile scissors is described in Eq. (18). The bilateral rolling shear consists 
of mobile and fixed scissors with synchronous kinematic velocities, $3 \#$ hydraulic cylinder whose movement rule is the same as that of $1 \#$ servo-cylinder, and $4 \#$ hydraulic cylinder whose movement rule is same as that of $2 \#$ servocylinder, which can be expressed as follows:

$\left[\begin{array}{c}S_{x}^{\prime} \\ S_{y}^{\prime} \\ \sin \varphi^{\prime} \\ \cos \varphi^{\prime}\end{array}\right]=\frac{1}{492804}\left[\begin{array}{ll}348.4 & -32.9 \\ 45.5 & 349.3 \\ 0.1 & -0.08 \\ 0.08 & 0.1\end{array}\right]\left[\begin{array}{c}y_{3}^{2} \\ y_{4}^{2}\end{array}\right]+\left[\begin{array}{c}0 \\ 0 \\ 0.11 \\ 0.99\end{array}\right]$

Where $S_{x}{ }^{\prime}$ and $S_{y}{ }^{\prime}$ are the horizontal and vertical displacements of upper blade for fixed scissors, respectively; and $y_{3}$ and $y_{4}$ are the displacements of $3 \#$ and $4 \#$ hydraulic cylinders for the fixed scissors, respectively.

According to the ideal cutting path, theoretical displacement of cylinders are developed by using the mathematical model established in this paper. So the pose of upper blade is automatically adjustable on line and the kinematics problem of the system is solved, which contribute to a deep understanding of control problems. What's more, to decrease horizontal displacement of blade arc and to increase evenness degree of blade overlapping is an important guarantee for the production of high quality steel plate shear section, so the orbit of the upper blade need to be adjusted to meet steel plates with different thickness.

\section{Analyzing the System Stability of the New Hydraulic Bilateral Rolling Shear}

Based on Eq. (18), the horizontal and vertical displacements of upper blade can be expressed as

$$
\left\{\begin{array}{l}
S_{x}=\frac{1}{492804}\left(348.4 y_{1}^{2}-32.9 y_{2}^{2}\right) \\
S_{y}=\frac{1}{492804}\left(45.5 y_{1}^{2}+349.3 y_{2}^{2}\right)
\end{array}\right.
$$

Take the derivative of both sides of Eq. (20) with respect to $y_{1}, y_{2}$ components

$$
\left\{\begin{array}{l}
\dot{S}_{x}=\frac{1}{492804}\left(696.8 y_{1} \dot{y}_{1}-65.8 y_{2} \dot{y}_{2}\right) \\
\dot{S}_{y}=\frac{1}{492804}\left(91 y_{1} \dot{y}_{1}+698.6 y_{2} \dot{y}_{2}\right)
\end{array}\right.
$$

Nonlinear state equation of system ${ }^{12-14)}$ are formulated

$$
f(y)=\frac{1}{492804}\left[\begin{array}{c}
696.8 y_{1} \dot{y}_{1}-65.8 y_{2} \dot{y}_{2} \\
91 y_{1} \dot{y}_{1}-698.6 y_{2} \dot{y}_{2}
\end{array}\right]
$$

The equilibrium state of the system known as in the initial position, namely, $f(0)=0$. The Jacobi matrix of system is

$$
F(y)=\frac{\partial f(y)}{\partial y^{\mathrm{T}}}=\left[\begin{array}{ll}
\frac{\partial f_{1}}{\partial y_{1}} & \frac{\partial f_{1}}{\partial y_{2}} \\
\frac{\partial f_{2}}{\partial y_{1}} & \frac{\partial f_{2}}{\partial y_{2}}
\end{array}\right]
$$

Sufficient condition for asymptotically stable of the system under equilibrium state is that $\hat{F}(y)=F^{\mathrm{T}}(y)+F(y)$ is negative definite matrix under any value of $y .{ }^{15)}$ In order to verify the conditions, detail derivation process as follows.

$$
\left.\begin{array}{r}
\hat{F}(y)=\frac{1}{492804}\left[\begin{array}{l}
1393.6\left(\dot{y}_{1}^{2}+y_{1} \ddot{y}_{1}\right) \\
91\left(\dot{y}_{1}^{2}+y_{1} \ddot{y}_{1}\right)-65.8\left(\dot{y}_{2}^{2}+y_{2} \ddot{y}_{2}\right)
\end{array}\right. \\
91\left(\dot{y}_{1}^{2}+y_{1} \ddot{y}_{1}\right)-65.8\left(\dot{y}_{2}^{2}+y_{2} \ddot{y}_{2}\right) \\
1397.2\left(\dot{y}_{2}^{2}+y_{2} \ddot{y}_{2}\right)
\end{array}\right] .
$$

Based on Sylvester criterion, ${ }^{16)}$ then

$$
\begin{aligned}
& \Delta_{1}=1393.6\left(\dot{y}_{1}^{2}+y_{1} \ddot{y}_{1}\right)<0 \\
& \Delta_{2}=|\hat{F}(y)|<0
\end{aligned}
$$

According to, ${ }^{17)}$

$$
\ddot{y}=1 / m_{t}\left(-k y-B \dot{y}+A_{1} P_{L}-F\right) .
$$

Substituting Eq. (27) into Eq. (25) is as below.

$$
\dot{y}_{1}^{2}+y_{1} \ddot{y}_{1}=\dot{y}_{1}^{2}+\frac{y_{1}}{m_{t}}\left(-k y_{1}-B \dot{y}_{1}+A_{1} P_{L}-F\right) . .
$$

In order to enjoy the constraint condition (25)

$$
\left(\dot{y}_{1}-B / 2 m_{t} y_{1}\right)^{2}<y_{1}\left[\frac{B^{2}+4 k m_{t}}{4 m_{t}^{2}} y_{1}-\frac{A_{1} P_{L}-F}{m_{t}}\right] . .
$$

Where, $B=300 \mathrm{~N} \cdot \mathrm{s} / \mathrm{m}, \mathrm{K}=20 \times 104 \mathrm{~N} / \mathrm{mm}, m_{t}=950 \mathrm{~kg}$, $A_{1}=360 \mathrm{~mm}^{2}, F=5.12 \times 106 \mathrm{~N} / \mathrm{mm}$.

So we can obtain as below.

$-\sqrt{210.55 y_{1}^{2}+5378.86 y_{1}}$

$+0.16 y_{1}<\dot{y}_{1}<\sqrt{210.55 y_{1}^{2}+5378.86 y_{1}}+0.16 y_{1}$

When $t=0.5 \mathrm{~s}, \quad y_{1}=23.36 \mathrm{~mm}, \quad f\left(y_{1}\right)_{\min }=$ $\sqrt{210.55 y_{1}^{2}+5378.86 y_{1}}+0.16 y_{1} \approx 494$ and $f\left(y_{1}\right)$ is a monotone increasing function when $0<y_{1}<288.48 \mathrm{~mm}$.

Speed of cylinder meets the demands: $-150 \mathrm{~mm} / \mathrm{s}<\dot{y}_{1}<150$ $\mathrm{mm} / \mathrm{s}$, obviously, Eq. (29) is correct, namely $\Delta_{1}<0$.

$$
a=\dot{y}_{1}^{2}+y_{1} \ddot{y}_{1}<0, \quad b=\dot{y}_{2}^{2}+y_{2} \ddot{y}_{2}
$$

Substituting Eq. (31) into Eq. (24) is as below.

$$
\hat{F}(y)=\left[\begin{array}{cc}
1393.6 a & 91 a-65.8 b \\
91 a-65.8 b & 1397.2 b
\end{array}\right]
$$

According to Eq. (26), the equation $\Delta_{2}=|\hat{F}(y)|<0$ is right under the hypothesis. So,

$$
\begin{aligned}
& |\hat{F}(y)|=1393.6 a \times 1397.2 b-(91 a-65.8 b)^{2} \\
& =(91 a-0.2 b)(215228.52 b-91 a)
\end{aligned}
$$

Where, $\mathrm{a}<0$ and $\mathrm{b}<0$, according to Eq. (26), $0.2 b<91 a<$ $21528.52 b$

Taking the integral of both sides of equation with respect to $y_{1}$ is as below

$$
\int 2.2 \times 10^{-3} b d y_{1}<\int a d y_{1}<\int 236.6 b d y_{1}
$$

Substituting Eq. (31) into Eq. (33) is as below.

$$
2.2 \times 10^{-3} b<\dot{y}_{1}<236.6 b
$$

Taking the integral of both sides of Eq. (34) with respect to $y_{2}$ is as below.

$$
2.2 \times 10^{-3} \dot{y}_{2}<\dot{y}_{1}<236.6 \dot{y}_{2}
$$


During the process of shearing plate, Eq. (35) meet the condition that $\Delta_{2}=|\hat{F}(x)|<0$.

So, $\hat{F}(x)$ is negative definite and the equilibrium state $y_{\mathrm{e}}=0$ for the system is asymptotically stable. In addition, when $\|y\| \rightarrow \infty$, we can get

$$
\begin{aligned}
& f^{\mathrm{T}}(y) f(y)=\left[\begin{array}{ll}
696.8 y_{1} \dot{y}_{1}-65.8 y_{2} \dot{y}_{2} & 91 y_{1} \dot{y}_{1}-698.6 y_{2} \dot{y}_{2}
\end{array}\right] \\
& {\left[\begin{array}{c}
696.8 y_{1} \dot{y}_{1}-65.8 y_{2} \dot{y}_{2} \\
91 y_{1} \dot{y}_{1}-698.6 y_{2} \dot{y}_{2}
\end{array}\right]=\left(696.8 y_{1} \dot{y}_{1}-65.8 y_{2} \dot{y}_{2}\right)^{2}} \\
& +\left(91 y_{1} \dot{y}_{1}-698.6 y_{2} \dot{y}_{2}\right)^{2} \rightarrow \infty
\end{aligned}
$$

So the system is global asymptotic synchronization at a state of equilibrium $y_{\mathrm{e}}=0$.

The same procedure is easily adapted to obtain behavior models for the Eqs. (18) and (19). The proving procedures are skipped.

The results suggest that the scheme meet the conditions of stability.

\section{Experiment Research}

In this study, the relationship between cylinder displacements and blade positions of the shearing mechanism is developed, which can make arcuate upper blade do movement of rolling shear. If mathematical model is not built as this article, then the shearing mechanism would be out of control, which leads to the problems that steel plate can not be cut off. Some defect such as rip can be found in the part of the plate as shown in Fig. 7. In this paper, the displacement of cylinder is given in every moment and closed-loop hydraulic servo system is applied to adjust the actual displacement of cylinder in real time. Then the trajectory of

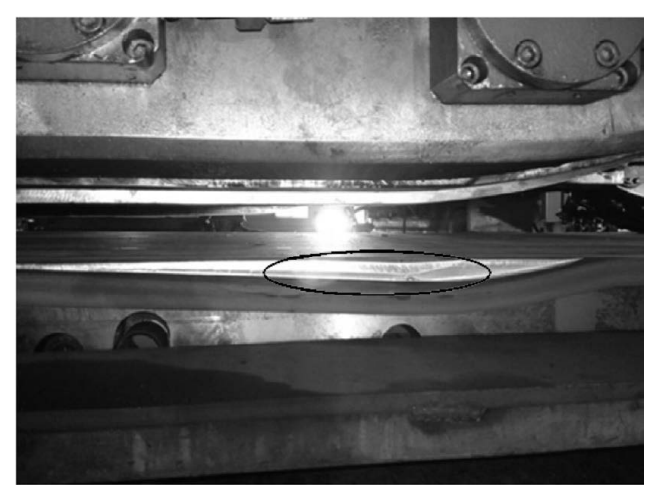

Fig. 7. Fracture surface.

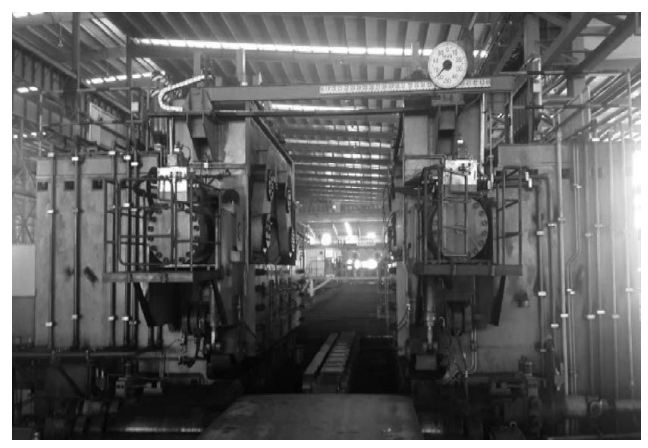

Fig. 8. Picture of the actual hydraulic bilateral shear. upper blade can keep the track of rolling shear trajectory, which can achieve an ideal motion and satisfy the shearing quality of steel plate.

\subsection{Experimental Procedures}

The new hydraulic bilateral rolling shear which is designed by the Taiyuan University of Science and Technology and produced by the Taiyuan Heavy Machinery Group, as shown in Fig. 8. A given numerical of the theoretical calculation need to be verified through the experiment, displacement is detected by the displacement sensor, which are both installed on the servo cylinder. The displacement of the upper blade cannot be directly measured, and thus, the displacement data of the cylinder are gathered.

\subsection{Experimental Results and Discussion}

The error between the actual and given displacements is analyzed by collecting the actual displacements of the four hydraulic servo cylinders. Comparing the actual and given displacements in the field. Firstly, 1\# and 3\# cylinders move at a specific speed; then, when it arrives at a certain point, 2\# and $4 \#$ right cylinders begin to move and enter the rollingcut stage to shear the plates. When $1 \#$ and $3 \#$ cylinders reach maximum displacement, i.e. $(288.72 \mathrm{~mm})$, it returns while $2 \#$ and $4 \#$ cylinders continue to extend $(224.83 \mathrm{~mm}$ is reached) until shearing is complete. Finally, the rods of the four cylinders return to their original positions. As shown in Fig. 9(a), the error of 1\# cylinder between the actual and given displacements is $0.55 \%$ to $15.3 \%$, that of $2 \#$ cylinder is $0.08 \%$ to $13.3 \%$ (Fig. $9(\mathrm{~b})$ ), that of $3 \#$ cylinder is $0.1 \%$ to $11.3 \%$ (Fig. 9(c)), and that of $4 \#$ cylinder is $0.4 \%$ to $5.8 \%$ (Fig. 9(d)). There is a gradual pressure for the upper blade in the process of cutting plate, so the error should be exist. The error is related to the thickness, the width and different materials of plate and so on. Therefore, this percentage can not be directly used as a standard to measure the precision of cutting mechanism. And shear quality of the plate is most relevant to the shear forces generated from the upper blade. According to the experience of the real experiment, the maximum shear force is 1.5 times then the theoretical value, the roll cutting angle range is $2^{\circ}-3^{\circ}{ }^{3)}$ According to reference, ${ }^{5)}$ cutting angle can be obtained, the maximum displacement error of hydraulic cylinder derived is 21.4 $\mathrm{mm}$. After analyzing test data collected in this paper, the maximum displacement error of hydraulic cylinder is not more than $3 \mathrm{~mm}$. So the precision of the cutting mechanism can be improved by controlling the displacement of the hydraulic cylinder, then the quality of steel plate can be improved. Both theoretical and field data indicate that the hydraulic servo control system can satisfy the shearing track characteristics of the hydraulic rolling shear.

The shearing mechanism does the movement as ideal trajectory and a strong shear force induced from cylinders, which will achieve the prediction accuracy. According to the ideal quality of the cross section, the required trajectory can be derived. As the mathematical model of the mechanism built in this paper, displacement of cylinders can be adjusted in real time, the upper blade can do the ideal rolling shear movement, which achieves the required cutting position accuracy and the quality of the cross section of plate steel as shown in Fig. 10. 


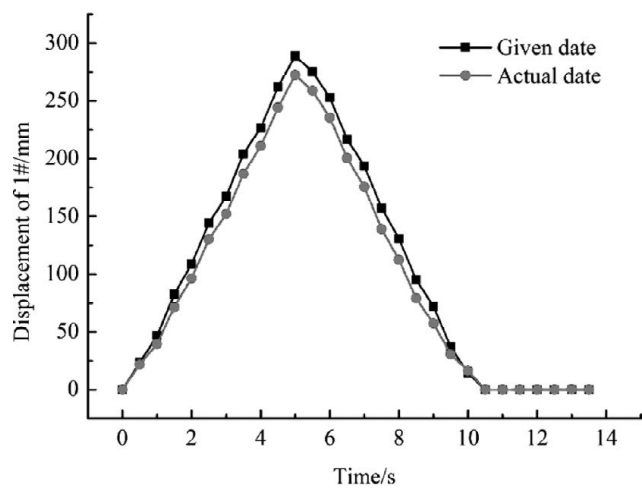

(a) The actual and given displacements of $1 \#$.

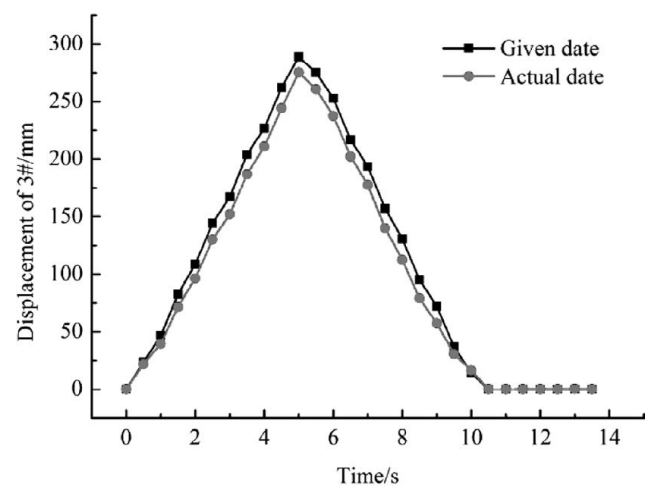

(c) The actual and given displacements of $3 \#$.

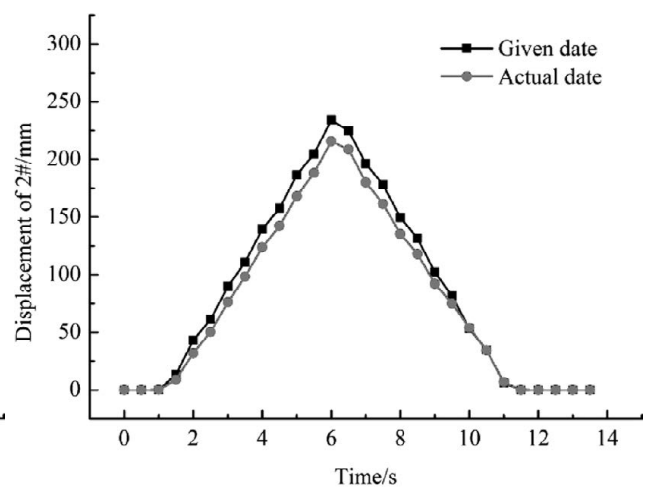

(b) The actual and given displacements of $2 \#$.

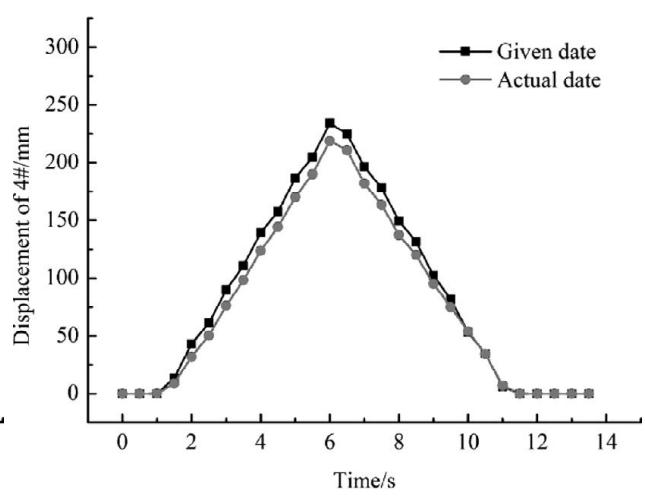

(d) The actual and given displacements of $4 \#$.

Fig. 9. The actual and given displacements of cylinders.

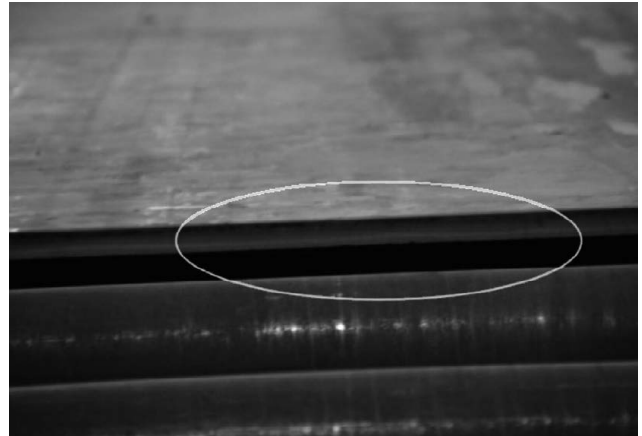

Fig. 10. The cross section of plate steel.

\section{Conclusions}

(1) In order to establish the mathematic model of new hydraulic bilateral rolling shear, the relationship of position and attitude of shearing mechanism is developed. Then the entire motion of upper blade can be expressed in the base coordinate system.

(2) In order to describe the various motions of upper blade, DOF of the mechanism is configured: the level movement toward the $\mathrm{x}$ axis direction, the vertical movement toward the $\mathrm{y}$ axis direction, and the rotation toward the $\mathrm{z}$ axis direction, and the general movement requirements are satisfied.

(3) In this study, the model of the mechanism is established based on the kinematics and inverse kinematics analysis. Moreover, by controlling the displacement of the hydraulic cylinder to adjust the position and attitude of the upper blade in real time, the stability of system and the quality of plant surface can be improved.

\section{Acknowledgements}

This project is supported by National Natural Science Foundation of China (Grant No. 51505315, 51375325, 51404160), Provincial Fund for Young Scholars (2013021019-2), the initial funding of doctor research of Taiyuan University of Science and Technology (20122047).

\section{REFERENCES}

1) Z. B. Chu, Q. X. Huang and L. F. Ma: J. Sichuan Univ.: Eng. Sci. Ed., 43 (2011), 247.

2) Y. G. Bondar, Y. N. Belobrov and A. A. Kalashnikov: Metallugist, 49 (2005), 456.

3) Q. X. Huang: Design of Rolling Mills, Metallurgy Industry Press, Beijing, (2007), 221, 261.

4) M. Murakawa and Y. Lu: J. Mater. Process. Technol., 66 (1997), 232.

5) R. Q. Zhang: The Research on Improving Thick Plates Shear Quality for Double Side Rolling Trimmer, Yanshan University, HeBei, (2008), 24.

6) H. Y. Han, Q. X. Huang, L. F. Ma and J. Wang: J. Sichuan Univ.: Eng. Sci. Ed., 43 (2011), 239.

7) L. F. Ma, Q. X. Huang, Y. Li, J. M. Wang and X. G. Wang: J. Sichuan Univ.: Eng. Sci. Ed., 40 (2008), 170.

8) L. F. Ma, Q. X. Huang, H. Y. Han, Z. B. Chu and Z. G. Li: J. Beijing. Univ. Technol., 38 (2012), 1601.

9) L. F. Jiang, T. Lan, Y. C. Zhang and G. Q. Ni: Proc. of SPIE-The Int. Society for Optical Engineering, OIT 2009, China, (2009), 7513.

10) S. W. Lee, K. S. Kwon and I. C. Park: IEEE T. Circuit.- II, 54 (2007), 680.

11) X. G. Ding: Research on Robotic Control, Zhejiang University Press, Hangzhou, (2006), 19.

12) Y. Wei: Liner Algebra Appl., 466 (2015), 102.

13) E. Defez, L. Jódar and A. Law: Compt. Math. Appl., 48 (2004), 789.

14) E. Fridman: Syst. Control. Lett., 43 (2001), 309.

15) K. M. Xie: Modern Control Theory, Tsinghua University Press, Beijing, (2012), 183.

16) N. Nakashima: Algebr. Represent Th., 17 (2014), 1163.

17) C. X. Wang: Hydraulic Control System, China Machine Press, Beijing, (2011), 40. 